\section{Case Reports in Oncology}

\title{
Cardiac Melanoma Metastasis with ERBB2 Gene Amplification: A Potential for Future Targeted Therapy
}

\author{
Polona Gams a, b Zvezdana Dolenc Stražar ${ }^{c} \quad$ Maja Šoštariča, b, d \\ Matic Bošnjakc Juš Kšelab, e \\ aDepartment of Anesthesiology and Intensive Care, Surgery Bitenc, Golnik, Slovenia; \\ bFaculty of Medicine, University of Ljubljana, Ljubljana, Slovenia; Institute of Pathology, \\ Faculty of Medicine, Ljubljana, Slovenia; ${ }^{d}$ Clinical Department of Anesthesiology \\ and Intensive Care, University Medical Centre, Ljubljana, Slovenia; ${ }^{\text {eDepartment of }}$ \\ Cardiovascular Surgery, University Medical Centre, Ljubljana, Slovenia
}

Keywords

Cardiac tumor $\cdot$ Melanoma $\cdot$ ERBB2 gene $\cdot$ HER2 gene $\cdot$ Trastuzumab

\begin{abstract}
Cardiac tumors are rare, and their treatment differs interindividually regarding the histopathological proprieties and the stage of disease. Authors present a case of symptomatic cardiac melanoma metastasis that expressed an ERBB2 (HER2) gene amplification in a course of the disease that has not yet been reported. The frail patient with a history of pulmonary and renal carcinoma, was admitted to the hospital due to a symptomatic left atrial tumor mass. The patient underwent a tumor-resecting cardiac surgery. At first mistaken for myxoma on echocardiography, the histopathological examination of the tumor revealed a melanoma of acral or mucosal origin. The melanoma metastasis was negative for common genetic mutations in $B R A F$, NRAS or KIT genes, and for the presence of NTRK genes fusions, but carried ERBB2 (HER2) gene amplification. The absence of standard gene mutations rendered it unresponsive to treatment with BRAF and MEK inhibitors. This molecular finding is rare in melanomas and represented a therapeutic target for off-label systemic treatment with drugs, primarily aimed at ERBB2 positive breast, gastric, and gastroesophageal junction cancers. A rare finding like this justifies molecular genetic analysis of unusual tumor specimen and guarantees optimal treatment for uncommon types of cardiac metastatic tumors.




\section{Introduction}

Cardiac tumors are rare. The incidence of primary heart tumors is $0.02 \%$, of which $75 \%$ are benign and $25 \%$ malignant. The most common primary tumor of the heart in adults is myxoma and in children rhabdomyosarcoma $[1,2]$. Metastases to the heart are 20-40 times more common than primary heart tumors [3]. They are most often diagnosed post-mortem due to lack of symptoms [4]. Their incidence in general population according to autopsy studies is $0.7-3.5 \%$ and increases up to $9.1 \%$ in patients with metastases from known noncardiac primary tumors. The most common metastases to the heart originate from lung cancer, breast cancer, and hematologic cancers. This is due to their relatively high incidence and aggressiveness. Spread to the heart can occur by different mechanisms, including direct growth (lung cancer), hematogenous (melanoma), retrograde lymphatic seeding (breast cancer), or venous extension (kidney cancer) [5]. Patients with a heart tumor often display non-specific symptoms that depend on the tumor location and the degree of tumor infiltration to neighboring tissues. Clinical picture varies from an incidental radiological finding to severe disease such as shock and sudden cardiac arrest. Diagnosis is usually confirmed by echocardiography, but can also be confirmed by CT or MR imaging [6].

Authors present a case report of symptomatic cardiac melanoma metastasis that carried a rarely observed $E R B B 2$ gene amplification. This is considered a positive predictive marker for systemic treatment with specific target drugs.

\section{Case Presentation}

A 77-year-old Caucasian woman with chronic kidney disease, diabetes mellitus type 2, heart failure, previous nephrectomy due to clear cell renal cell carcinoma, and previous upper right lobectomy of the lung due to adenocarcinoma was admitted to the hospital because of acute hypercapnic respiratory insufficiency. She needed respiratory support with noninvasive ventilation. She was also hemodynamically unstable.

CT angiography of the pulmonary arteries was performed. The CT scan revealed leftsided subsegmental pulmonary thromboembolism and a suspicious mass in the left atrium. She was treated for pulmonary embolism. The previous CT scan of her thorax, which she had undergone a couple of months before for her pulmonary carcinoma follow-up, had shown a normal image of the heart, proving the mass in the left atrium grew de novo. The transthoracic and transesophageal echocardiography discovered a massively enlarged left atrium, fibrous changes of the mitral valve and a mobile tumor formation measuring $2.8 \times 3.6 \mathrm{~cm}$ that arose from the posterior annulus (shown in Fig. 1).

The stem of the discovered formation was broad but did not reach the leaflet of the mitral valve. The tumor prolapsed through the mitral valve and caused severe mitral stenosis with minimal regurgitation. The tumor seemed to have caused postcapillary pulmonary hypertension as well. Cardiologists suspected it was myxoma and referred the patient to cardiac surgeons. A preoperative PET scan showed high metabolic activity in the left atrium that pointed to the possibility of the tumor's more aggressive origin, such as metastasis or a primary malignant tumor of the heart rather than myxoma. The patient underwent an open mitral valve replacement and extirpation of the tumor of the left atrium. At the time of openheart inspection, the tumor was not encapsulated, but grew inside the atrial wall with rootlike extensions. It hung on a stem that masked its appearance as myxoma on echocardiography. It did not overgrow the mitral valve (shown in Fig. 2a). Surgeons reconstructed the left atrial wall and replaced the diseased mitral valve with an artificial biological valve.

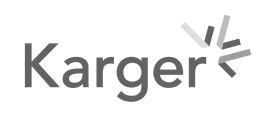



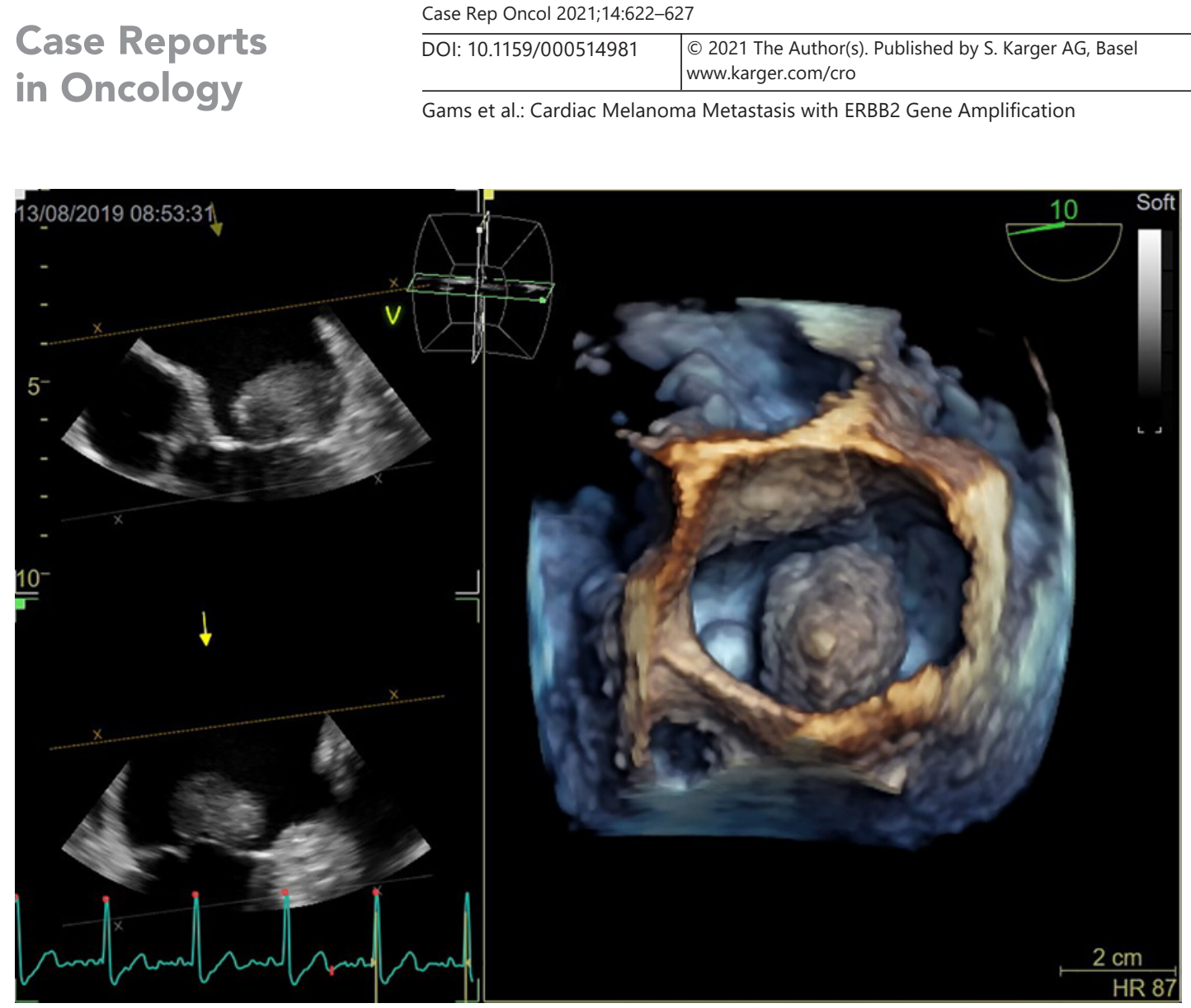

Fig. 1. Echocardiographic image, 2D (left) and 3D (right). A tumor mass, suspected for myxoma is visible in the left atrium. Reproduced with kind permission from Prof. Mojca Bervar.

A histopathological examination (shown in Fig. 2b-d) revealed a pleomorphic spindlecell malignant tumor with high mitotic activity and focal necrosis. Immunohistochemically, the tumor cells were diffusely positive for S100 protein, SOX10, PRAME and focally positive for melan A and melanoma cocktail, negative for HMB45, CD31, CD34, ERG, desmin, GMA, Myf4, h-Caldesmon, calponin, CK18, CKAE1AE3, DOG1, EMA, STAT6, PAX8, MITF, GFAP, consistent with diagnosis of metastatic melanoma.

Molecular genetic analysis of the tumor did not confirm the presence of common genetic mutations in melanoma BRAF, NRAS or KIT, or the presence of NTRK genes fusions, but confirmed the presence of ERBB2 (HER2) gene amplification.

The patient experienced several anticipated postoperative complications, including atrial fibrillation, pulmonary edema with respiratory failure and deterioration of a preexisting kidney disease. Following a full recovery, she was discharged from the hospital and referred to designated cancer center for further oncological treatment. Sadly, she passed away before systemic treatment initiation. The primary source of melanoma was not identified.

\section{Discussion/Conclusion}

The patient with symptomatic cardiac tumor was indicated for surgery of myxoma due to its appearance on echocardiography. A pedunculated tumor, arising from the septum or posterior wall of the left atrium, perhaps even prolapsing across the mitral valve, seen on 


\section{Case Reports in Oncology}
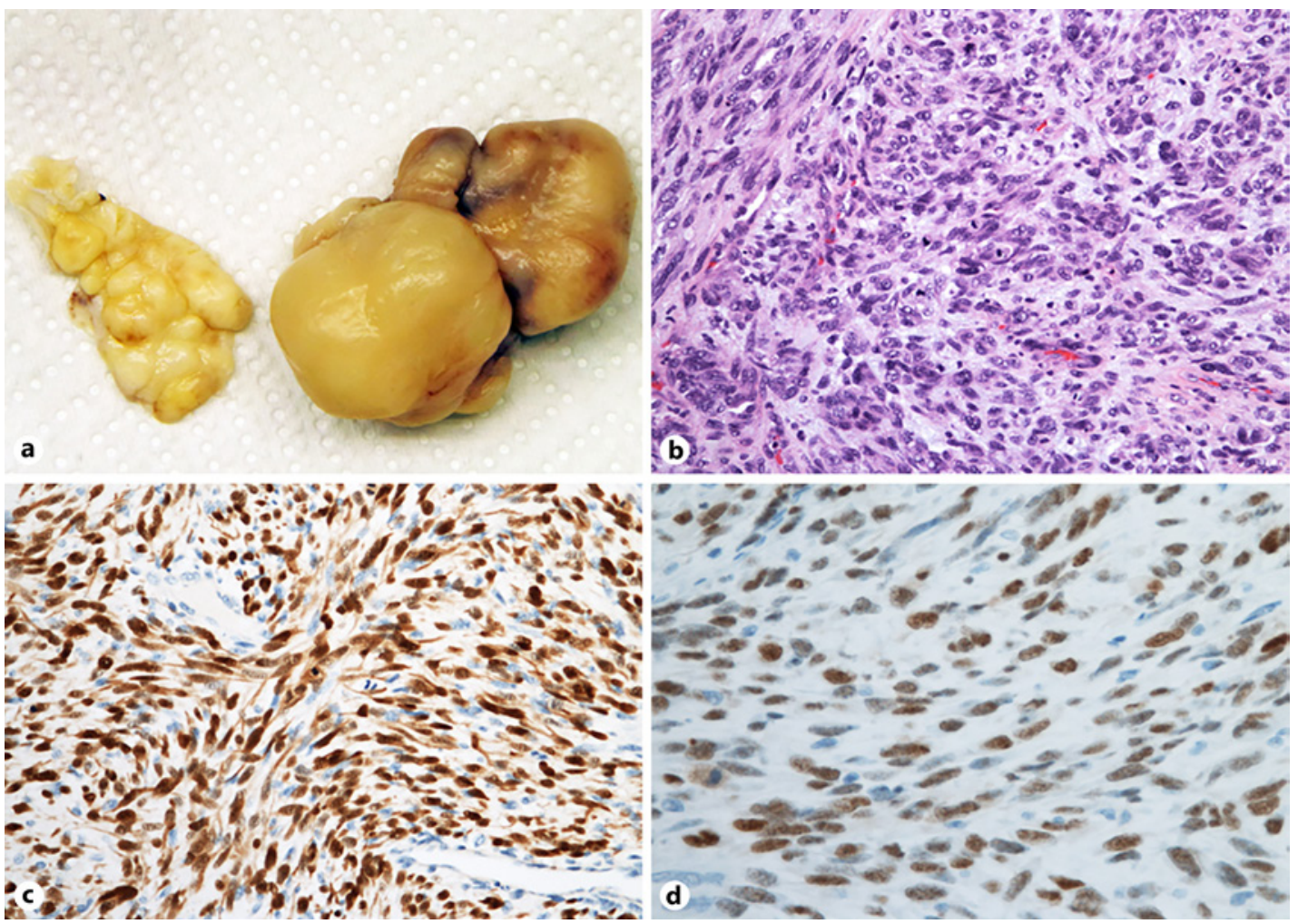

Fig. 2. a The protruding pedunculated part of the left atrial malignant melanoma metastasis, situated above the mitral valve and growing into the interatrial septum. b Pleomorphic spindle cells with prominent nucleoli and high mitotic activity, arranged in random-oriented short fascicles. HE staining, $\times 200$. c Strong diffuse nuclear and cytoplasmic staining for S100 protein, $\times 400$. d Strong diffuse nuclear staining with PRAME, $\times 400$.

diagnostic imaging, is more characteristic for myxoma than a cardiac metastasis [7]. Only the histopathological examination revealed that it was a metastatic melanoma to the heart.

Metastatic melanoma has poor prognosis with a median survival time of only 9 months and $10 \%$ long-term survival rate [8]. Resection of systemic, especially solitary melanoma metastases, may increase patient survival. This was proven for pulmonary metastatic disease, where the 5-year survival after metastasectomy is 39\% [9]. Surgical resection of secondary heart tumors is feasible only in selected patients and usually requires additional neoadjuvant therapy $[10,11]$.

There are several methods of systemic treatment of melanoma including chemotherapy with cytokines such as interleukin-2 and interferon-alpha, immune checkpoint inhibitors like anti-CTLA4 and anti-PD1 monoclonal antibodies and small-molecule targeted drugs used for melanomas with specific gene mutations [12]. The current most effective targeted treatment according to the Cochrane review from 2018 is with $B R A F$ and $M E K$ inhibitors. These small molecule-targeted drugs are only efficient for tumors positive for the presence of $B R A F$ and $M E K$ mutations.

ERBB2 amplification is present in a minority of melanomas [13]. According to a study of 600 melanoma specimens, the ERBB2 amplification is present in $3.6 \%$ of recurrent or metastatic melanomas [14]. It encodes the HER2 receptor, which is a tyrosine-kinase receptor and a tumor factor from a superfamily of EGFR markers that controls growth, survival, adhesion and migration of the cells. It follows an intracellular signal pathway that controls enhancement 
or suppression of the cell [15]. Mutations in the ERBB2 gene that carry a gene transcription for HER2 receptors, can lead to uncontrolled cell proliferation and oncogenesis. Amplification of the ERBB2 is seen in rare cases of acral or mucosal melanomas and is not present in any other type of skin melanoma. This can be used as aid in localizing the primary tumor. The $E R B B 2$ gene amplification is a novel target for systemic treatment and is best understood for the management of breast adenocarcinoma, where it can be treated by HER2-directed monoclonal antibodies (trastuzumab and pertuzumab), antibody-drug conjugates (trastuzumab emtansine), and HER2 kinase inhibitors (lapatinib) $[13,16]$. Trastuzumab is approved also for gastric and gastroesophageal cancer [17]. The ERBB2 gene-targeted therapy is used as neoadjuvant therapy for patients with other solid and systemic tumors including bladder, small bowel, ampullar, skin non-melanoma and cervical cancer $[18,19]$.

The patient presented in this case was in frail general condition and passed away shortly after completing the surgical part of treatment. According to genetic analysis, systemic treatment with $E R B B 2$ gene amplification-targeted drugs like trastuzumab, pertuzumab, or lapatinib would likely warrant positive results. This finding justifies molecular genetic analysis of unusual tumor specimen and guarantees optimal treatment for uncommon types of cardiac metastatic tumors in future patients.

\section{Statement of Ethics}

Written informed consent was obtained from the patient for publication of this case report and any accompanying images.

\section{Conflict of Interest Statement}

The authors have no conflict of interest to declare.

\section{Funding Sources}

The manuscript has not been funded by any party.

\section{Author Contributions}

The authors P.G., Z.D.S., M.Š., M.B., and J.K. have substantially contributed to conception of the work, revised it critically for important intellectual content, approved the final version to be published and agreed to be accountable for all aspects of the work.

\section{References}

1 Hoffmeier A, Sindermann JR, Scheld HH, Martens S. Cardiac tumors - diagnosis and surgical treatment. Dtsch Arztebl Int. 2014;111(12):205-11.

2 Di Vito A, Mignogna C, Donato G. The mysterious pathways of cardiac myxomas: a review of histogenesis, pathogenesis and pathology. Histopathology. 2015;66(3):321-32.

3 Del Carpio Huerta LP, Mas Castells M, Anguera Palacios G, Sullivan I, Gonzalez Vidal A, Majem Tarruella M. A novel KRAS mutation in metastasic conjunctival melanoma: a case report and literature review. Melanoma Res. 2018;28(2):79-81.

\section{Karger'}


4 Villa A, Eshja E, Dallavalle S, Bassi EM, Turco A. Cardiac metastases of melanoma as first manifestation of the disease. J Radiol Case Rep. 2014;8(4):8-15.

5 Maleszewski JJ, Bois MC, Bois JP, Young PM, Stulak JM, Klarich KW. Neoplasia and the heart: pathological review of effects with clinical and radiological correlation. JACC cardiooncol. 2018;72:202-27.

6 Abbas A, Garfath-Cox KA, Brown IW, Shambrook JS, Peebles CR, Harden SP. Cardiac MR assessment of cardiac myxomas. Br J Radiol. 2015;88(1045):20140599.

7 Jain S, Maleszewski JJ, Stephenson CR, Klarich KW. Current diagnosis and management of cardiac myxomas. Expert Rev Cardiovasc Ther. 2015;13(4):369-75.

8 Turner N, Ware 0, Bosenberg M. Genetics of metastasis: melanoma and other cancers. Clin Exp Metastasis. 2018;35(5-6):379-91.

9 Younes R, Abrao FC, Gross J. Pulmonary metastasectomy for malignant melanoma: prognostic factors for longterm survival. Melanoma Res. 2013;23(4):307-11.

10 Yanagawa B, Mazine A, Chan EY, Barker CM, Gritti M, Reul RM, et al . Surgery for tumors of the heart. Semin Thorac Cardiovasc Surg. 2018 Winter;30(4):385-97.

11 Maverakis E, Cornelius LA, Bowen GM, Phan T, Patel FB, Fitzmaurice S, et al. Metastatic melanoma - a review of current and future treatment options. Acta Derm Venereol. 2015;95(5):516-24.

12 Pasquali S, Hadjinicolaou AV, Chiarion Sileni V, Rossi CR, Mocellin S. Systemic treatments for metastatic cutaneous melanoma. Cochrane Database Syst Rev. 2018;2:CD011123.

13 Gottesdiener LS, O'Connor S, Busam KJ, Won H, Solit DB, Hyman DM, et al. Rates of ERBB2 alterations across melanoma subtypes and a complete response to trastuzumab emtansine in an ERBB2-amplified acral melanoma. Clin Cancer Res. 2018;24(23):5815-9.

14 Kluger HM, DiVito K, Berger AJ, Halaban R, Ariyan S, Camp RL, et al. Her2/neu is not a commonly expressed therapeutic target in melanoma -- a large cohort tissue microarray study. Melanoma Res. 2004;14(3):207-10.

15 Jeon HJ, Choi BBR, Park KH, Hwang DS, Kim UK, Kim GC. Induction of melanoma cell-selective apoptosis using anti-HER2 antibody-conjugated gold nanoparticles. Yonsei Med J. 2019;60(6):509-16.

16 Ahmed S, Sami A, Xiang J. HER2-directed therapy: current treatment options for HER2-positive breast cancer. Breast Cancer. 2015;22(2):101-16.

17 Meric-Bernstam F, Johnson AM, Dumbrava EEI, Raghav K, Balaji K, Bhatt M, et al. Advances in HER2-targeted therapy: novel agents and opportunities beyond breast and gastric cancer. Clin Cancer Res. 2019;25(7):203341.

18 Cousin S, Khalifa E, Crombe A, Laizet Y, Lucchesi C, Toulmonde M, et al. Targeting ERBB2 mutations in solid tumors: biological and clinical implications. J Hematol Oncol. 2018;11(1):86.

19 Franchino F, Rudà R, Soffietti R. Mechanisms and therapy for cancer metastasis to the brain. Front Oncol. 2018; 8:161. 